\title{
Discussion on the TCM Diagnosis and Treatment Ideas and Methods of Short Stature from the Theory of "Spleen-Liver-Kidney Correlation"
}

\author{
Lu Xia', Hui Zhang ${ }^{2, *}$, Xuan Jin², Yanfang $\mathrm{Ma}^{2}$ \\ ${ }^{1}$ Shaanxi University of Chinese Medicine, Xianyang, Shaanxi, 712000, China. \\ ${ }^{2}$ Affiliated Hospital of Shaanxi University of Chinese Medicine, Weiyang West Road, Xianyang, Shaanxi, 712000, China.
}

How to cite this paper: Lu Xia, Hui Zhang, Xuan Jin, Yanfang Ma. (2020) Discussion on the TCM Diagnosis and Treatment Ideas and Methods of Short Stature from the Theory of "Spleen-Liver-Kidney Correlation". International Journal of Clinical and Experimental Medicine Research, 4(4), 248-253.

DOI: 10.26855/ijcemr.2020.10.021

Received: October 13, 2020

Accepted: November 8, 2020

Published: November 17, 2020

*Corresponding author: Hui Zhang, Affiliated Hospital of Shaanxi University of Chinese Medicine, Weiyang West Road, Xianyang, Shaanxi, 712000, China.

Email: 405508885@qq.com

\begin{abstract}
Idiopathic short stature is the most common type of short stature, and the cause of modern medicine is unknown. Professor Zhang Hui based on years of clinical treatment experience, it is believed that the cause of this disease is closely related to congenital factors, eating disorders, and emotional disorders. The disease is located in the spleen, liver, and kidneys. When the spleen and stomach are dystrophic, it is easy to aggravate stagnation of liver qi, weak kidney qi, and loss of qi and blood in the five organs. And, the body fluid infusion is abnormal and the body loses nourishment, resulting in growth retardation. Combined with the physiological and pathological characteristics of the child's spleen deficiency, the spleen and stomach are easy to be injured, and it is easy to transfer to the viscera. Therefore, the spleen is often the center in the treatment process. According to the degree of spleen deficiency, Professor Zhang Hui divided the disease into three types: mild, medium, and severe, and supplemented with 1 medical record, confirming that the spleen is the core and the liver and kidney coordinating therapy is quite effective and worthwhile Clinical reference.
\end{abstract}

\section{Keywords}

Idiopathic short stature, spleen-liver-kidney related theory, spleen deficiency

Diagnosis and treatment of idiopathic short stature (Idiopathic Short Stature, ISS) in Chinese medicine refers to children with short stature whose etiology is not yet clear and there is no pathological cause. When healthy children of the same race, gender, age, and similar environment are used as a reference standard, their height is 2 standard deviations (-2SD) below the average or below the 3rd percentile [1]. According to relevant research statistics, ISS is the most common type of children with short stature in my country, accounting for about $50 \%$ of children with short stature [2]. ISS not only affects the life-long height of children, but also has a more profound negative impact on children's psychological behavior and social life [3]. At present, the main clinical drug used to treat ISS is genetically recombinant human growth hormone (rhGH), but the current use of rhGH to treat ISS is not listed in the main instructions for rhGH, and the use of rhGH to treat ISS has been quite controversial. On the one hand, its price is expensive, painful and relatively inconvenient to operate. On the other hand, long-term use of rhGH therapy may induce tumors and affect glucose metabolism [4-5]. Therefore, the clinical advantages of traditional medicine can be used in the treatment of ISS, and the combination of traditional Chinese and western medicine can provide a safer, cheaper and effective method for ISS. Chief Physician Zhang Hui has been working in pediatrics of tradition- 
al Chinese medicine for more than 30 years. She is good at treating children's endocrine system diseases, especially in the treatment of ISS with integrated Chinese and Western medicine. The author is fortunate to learn from Professor Zhang Hui and follow up with doctors. The spleen is the core, collecting, combing, and summarizing the experience of the "spleen-liver-kidney related" theory in the treatment of ISS to provide new ideas for the clinical treatment of ISS.

\section{With the spleen as the core, the theoretical origin of the liver and kidney}

Zhu Zhenheng's "Danxi Heart Method” says: “The reason why the people has all her life is water and valley. Water is the kidney and the valley is the spleen",Water valley is the basic substance to maintain the normal functions of the human body, and the kidney and spleen are the masters of water and valley respectively, so the spleen and kidney maintain the basic functions of the human body. "The kidney is the foundation of the congenital, and the spleen is the foundation of the acquired". The congenital kidney essence depends on the nourishment of the water and valley essence of the acquired spleen. If the congenital essence is insufficient or the acquired spleen is abnormal, it is easy to provide nourishment to the various organs. Growth retardation occurs.

On the other hand, "earth is derived from wood". Soil relies on the dredging of liver wood. In terms of blood circulation, the spleen controls the blood, and the liver controls the blood. Only when the temper is strong, the qi and blood can continuously nourish the liver qi and make the liver. There is a degree of concealment and drainage, and the organization is smooth; the liver and blood are sufficient to make the spleen biochemically active and have blood. If the spleen or liver is abnormal, the source of qi and blood is depleted, and the five internal organs are insufficiently moisturized, which is prone to growth retardation.

From the perspective of modern medicine, theories such as "gut-brain axis", "gut-liver axis" and "gut-mandrel axis" have gradually appeared in the field of vision of many scholars. The emergence of these theories no longer connects the various systems of the human body. Treat independently, but link two or more systems into new functional units. The two or more interact and adjust through the "axis". These doctrines once again unite the human body organically from the micro to the macro. The concepts of "holistic concept" and "correlation of five internal organs" in Chinese medicine coincide, especially the "gut-brain axis" and "gut-liver axis" are related to the theory of "spleen and kidney related" and "liver and spleen related" theory in Chinese medicine. The similarity is extremely high. Intestinal microbes can achieve two-way regulation through the "gut-brain axis" and "gut-liver axis". From the perspective of Chinese medicine, the kidney is connected to the brain, and the "spleen-kidney related" can also be understood as the relationship between the spleen and the kidneys and brain. The functions of each other are closely related [6-7]. The theories of "spleen and kidney related" and "liver and spleen related" suggest that the viscera are physiologically interconnected and mutually beneficial, and pathologically transmitted to each other. The "spleen-related" theory does not only refer to the relationship between the liver, spleen, and kidney, but rather emphasizes the mutual influence of their functions [8-10].

Based on this theory, Chief Physician Zhang Hui discovered that the spleen (intestinal microbes) was used as a hub to connect with other viscera (systems) when treating short stature, and the concept of liver and kidney coordinating was well applied. After years of clinical analysis, summarize, and finally form the "spleen-liver-kidney correlation" theory, and apply this theory to the guidance of the etiology and pathogenesis of ISS and the treatment of this disease.

\section{2 .The "spleen-liver-kidney-related" theory guides the etiology and pathogenesis of ISS}

The etiology and pathogenesis of ISS are still unclear in ancient and modern times. The analysis and summary of the etiology of ISS in modern medicine mainly include three factors: genetic factors, neuroendocrine factors and environmental factors [11]. Professor Zhang Hui combined with the physiological characteristics of children's "spleen often insufficiency and kidney often insufficiency" and believed that the disease is located in the spleen, liver, and kidneys. Teacher Zhang believes that the disease is not only responsible for one of the three organs, but is Congenital deficiency of kidney qi, acquired dystrophy of the spleen and stomach, and stagnation of liver qi are the main three causes of qi and blood loss in the five internal organs, abnormal fluid infusion, loss of body nourishment, and ultimately growth retardation, of which spleen deficiency is the core.

Insufficient kidney qi, acquired dystrophy of the spleen and stomach, Chinese medicine believes that "essence" is one of the basic substances that are born with and constitute the human body. "Two gods fight each other and form together, and they are often born before the body is called essence". "Lingshu· Juqi", this is the best interpretation of the source of "essence". The human body's "essence" can be divided into two parts: the innate essence inherited from the parents and the acquired essence transformed from the internal organs. Enclosed in the kidney, on the one 
hand, the abundant kidney essence can promote the growth and development of the human body and the maturity of reproductive function. On the other hand, the kidney also controls the bones to generate marrow. The abundant kidney essence can promote the production of bone marrow, and the bones have bone marrow. A steady stream of nourishment can develop normal, tough and powerful.

The filling of the essence of the kidney is the key to determining the growth and development of childhood. The innate endowment refers to the innate essence inherited from the parents. If the innate endowment is insufficient, it will cause the child's kidney essence weakness syndrome, which manifests as fetal cowardice in infancy. If it cannot be corrected in time, it will cause skeletal growth retardation, delayed growth and development and even mental retardation in childhood. With the advancement of modern medicine, test-tube babies, twins or multiple fetuses, advanced mothers, second fetuses, and small-for-gestational-age babies are more common. ISS caused by these reasons should belong to the syndrome of kidney weakness caused by insufficient congenital endowment. Although congenital factors dominate the growth and development of children, and studies have shown that genetic factors have an impact on height of about 70\% [12], the congenital essence depends on the acquired intensive nutrition. The syndrome of kidney weakness is further aggravated, and it can be seen that whether the acquired essence is full or not is the dominant position in this disease.

Acquired improper feeding, liver qi stagnation in the inner spleen is located in the middle Jiao, which is the foundation of acquired. The five elements belong to the soil, and the soil is called Jiahu. It has the properties of biochemistry, bearing, and acceptance. The spleen governs the movement and transformation, and is the source of qi and blood. People's daily intake of water grains decomposes into the stomach and then passes through the small intestine to clear the turbidity, and finally produce water grains and other substances. These water grains are dispersed to the internal organs through the spleen's transport and transformation function. Its function is described as follows: "People receive qi from the valley, and the valley enters the stomach to pass it through the lungs. All the internal organs and six organs are to receive qi...”. Children belong to a special group with the physiological characteristics of "infant yin and juvenile yang" and "inadequate spleen". With the advent of 2020, China has entered a well-off society in an all-round way. Compared with ancient children, due to lack of milk and food, qi and blood are biochemical deficiency of the spleen qi caused by lack of source is more likely to have the syndrome of liver depression and spleen deficiency with the characteristics of the times. There are two main reasons for the syndrome of liver depression and spleen deficiency: 1 . People who eat too much, the spleen is also weak. In children, a series of improper regulation reasons such as overfeeding, addiction to fat and sweet taste, gluttony and cold drinks, and less exercise, make the spleen and stomach relatively inferior to the function of the spleen and the stomach, which leads to the loss of the spleen and stomach. Abnormal lifting and lowering, dampness and turbidity endogenous for a long time, gathering into phlegm, forming the syndrome of phlegm-dampness trapping the spleen; 2 . With the increasing awareness of competition in modern society, children start to face competition and learn from the preschool period (over 3 years old) Increasing pressure and decreasing relative exercise time. On the basis of the syndrome of phlegm-dampness trapping the spleen, there will inevitably appear the syndrome of thinking about hurting the spleen. Excessive thinking will damage the spleen. In addition, prolonged depression can easily cause stagnation of liver qi and dysfunction of dredging. It affects its ability to control bile excretion and promote gastrointestinal digestion. On the other hand, liver dysfunction affects the patency of the whole body's qi and cannot effectively coordinate the spleen's transport and transformation functions. At this time, the spleen soil is already weak and the liver tree multiplies it, making the original the weakened spleen soil's transport and transformation function is even worse, and even tually the syndrome of liver depression and spleen deficiency is formed. Professor Zhang Hui concluded that in the growth and development of children, the three organs of liver, spleen, and kidney interact with each other physiologically and influence each other pathologically. The spleen should occupy a dominant position in the diagnosis and treatment of ISS. Spleen deficiency is aggravated and caused Kidney weakness and liver depression are the roots. Therefore, whether it is the syndrome of kidney weakness caused by inadequate endowment or the syndrome of liver depression and spleen deficiency caused by improper care, the core pathogenesis of ISS is spleen deficiency. When correcting ISS, the main contradiction should be grasped. The treatment of the spleen should be the primary task of this disease. The main treatment is to replenish the spleen, invigorate the spleen, and promote the spleen. Through the mutual use of the spleen-liver and kidney, the purpose of invigorating the kidney, regulating the liver and promoting the growth and development of children is achieved. This is the explanation of the etiology and pathogenesis of idiopathic short stature in children by the theory of "spleen-liver-kidney correlation”. 


\section{Guidance on the classification of ISS from the theory of "spleen-liver-kidney correlation" and the selection of prescriptions and medications}

Based on the theory of "spleen-liver-kidney correlation”, Mr. Zhang proposed the treatment of ISS based on the condition of the disease according to the relationship between the degree of spleen deficiency and liver and kidney. There are three types: weak spleen, spleen deficiency, liver depression, and liver depression and kidney weakness:

(1) Weak spleen: this type of child has no symptoms of congenital insufficiency, symptoms of spleen deficiency are mild, and the child is relatively backward in stature compared with children of the same age, and the complexion is pale yellow. The spirit is fair, does not like exercise, poor appetite, full after eating or the child eats a lot but short stature, pale red tongue, thin white coating, slow pulse, this type of child is mainly syndrome of spleen-qi deficiency, during treatment Commonly used drugs: Radix Atractylodes, Atractylodes macrocephala, Poria cocos, Tangerine peel, Amomum villosum, Atractylodes atractylodes, Magnolia officinalis, Licorice, etc. If the child has symptoms of liver depression, such as uncomfortable mood, depression or irritability, etc., Bupleurum, Cyperus rotundus, bergamot and other drugs for soothing liver and qi can be added; if the child has severe spleen deficiency and dampness, increase the amount of tangerine peel, and add Pinellia to strengthen the dampness and expectorant effect; if the child has poor appetite, For easy food accumulation, Jiao Sanxian, Gallus gallus domesticus, and Citrus aurantium can be added to strengthen the elimination of accumulation and stagnation, and promote the function of spleen and stomach transportation and transformation.

(2) Spleen deficiency and liver depression type: This type of children has no obvious congenital insufficiency, but this type of spleen deficiency is further aggravated on the basis of the weak spleen and also has symptoms of liver depression. It is manifested as the child's body development is lagging behind the children of the same age, and the complexion is chlorosis or pale, poor spirit, lack of exercise, small diet, partial eclipse, or large appetite, the body looks strong but the muscles are loose and weak, the emotional thinking is too good or the irritable, the concentration is difficult, the academic performance is poor ideal, poor night rest, pale tongue, white fur, thin pulse strings, this type of child has spleen deficiency and liver depression, commonly used drugs for treatment: peony, atractylodes, tuckahoe, tangerine peel, peony, chuanxiong, chaihu, citronella, incense Supplements, scutellaria, roasted licorice, etc. If the spleen-deficiency symptoms of children are severe, add astragalus, lentils, yam and other spleen and nourishing products on the basis of Sijunzi Decoction. If the liver depression has the potential to reduce fire and damage yin, it can be used here on the basis, the medicines for clearing away heat and nourishing yin, such as paeonol, red peony root, medlar, dendrobium, etc. are used to sooth the liver and relieve depression while preventing liver depression and transforming fire.

(3) Spleen deficiency and kidney weakness type: children with congenital deficiency or (and) growth retardation caused by acquired dystrophy belong to this type. This type of child is the most severely ill, manifested as the child is absolutely lagging behind the same age, and the complexion is chlorosis or pale , lack of energy, lack of energy, lazy speech, lean body, obvious muscle relaxation, lack of food or even no eating, some children usually addicted to cold drinks, snacks, etc., weak waist and knees, mental fatigue, pale tongue, white fur, weak pulse This type of child has both spleen and kidney deficiency, whether it is due to congenital deficiency, acquired loss of nutrition, or both causes, the child should be treated as a spleen and kidney, commonly used drugs: Pao Fuzi, dried ginger, Codonopsis, Baizhu, Gui Branches, eucommia ulmoides, roasted licorice, etc., if the child has short stature, waist and knee weakness caused by insufficient true yin, deficiency of the essence, etc., flesh and blood products such as tortoise shell and antler glue should be added on the basis of this prescription to fill the essence. The marrow is combined with achyranthes, rehmannia, yam and other medicines to nourish yin and replenish essence; if the child has symptoms of kidney-yang deficiency such as cold limbs, lack of energy, etc., cinnamon, eucommia, antler gum, etc. should be added to this prescription. Isothermal products for nourishing kidney-yang.

\section{Case examples of medical records}

Li, male, date of birth: November 28, 2013, first visit on May 26, 2018.

Main complaint: Found to grow slowly for 1 year.

History of present illness: Since the child entered kindergarten a year ago, it has been found that the growth and development is slower than that of children of the same age. The annual growth is about $3-4 \mathrm{~cm}$. The height before entering the kindergarten is about $94 \mathrm{~cm}$, and the appearance height is $97 \mathrm{~cm}$. I went to an outside hospital 1 year ago and performed liver function, kidney function, thyroid function, serum vitamin D, insulin-like growth factor-1, growth hormone provocation test, head MRI, bone age and other examinations without obvious abnormalities. The outside hospital diagnosed. "Idiopathic short stature", it is recommended to use rhGH treatment. After the family 
members refuse to give the child oral "calcium gluconate oral liquid, vitamin D drops" and other drugs for 1 year, no obvious curative effect is seen. I am now seeking Chinese medicine for diagnosis and treatment. The child is usually prone to dryness and irritability. He likes to eat beef and mutton, cold drinks and puffed foods. The amount of meals is small. After meals, he often complains of abdominal distension, abdominal pain, difficulty falling asleep, dry stool, one line for two days, and normal urination. Previously healthy, denying history of genetic diseases, thyroid function diseases and other diseases.

Personal history: G2P2, full-term normal delivery, artificial feeding, supplementary food for 10 months, birth weight $3.25 \mathrm{~kg}$, birth length $51 \mathrm{~cm}$.

Family history: Both his parents are healthy, his father is $180 \mathrm{~cm}$ tall, his mother is $162 \mathrm{~cm}$ tall, and his target's height is $177.5 \mathrm{~cm}$.

Physical examination: thin body, height: $97 \mathrm{~cm}$, weight: $14 \mathrm{~kg}$, no secondary sexual characteristics development.

Auxiliary examination: bone age (2018-5-26 outpatient of our hospital): in line with the bone age of 5-year-old children.

The symptoms are: thin body, poor spirits, complexion chlorosis, sparse hair color yellow and no luster, slightly swollen eyes, muscle atrophy of limbs, pale tongue, white fur, thin pulse.

Diagnosis: Western medicine diagnosis: idiopathic short stature. Chinese medicine diagnosis: five delays, five soft diseases (liver stagnation and spleen deficiency syndrome).

Treatment: invigorating the spleen and soothing the liver, replenishing qi and nourishing yin. Prescription: Sijunzi Decoction and Chaihu Shugan Powder. The specific prescriptions are as follows:

Codonopsis 10 g Atractylodes Rhizome 10 g Poria 10 g Roasted Licorice 6 g Jiao Sanxian 10 g each 10 g 28 doses, decocted in water, two doses a day. In addition, in cooperation with the growth and development of children in our hospital, two times a week, the family members are instructed to give the children a light and easy-to-digest diet, reduce the intake of snacks and cold drinks, strengthen the children's exercise, and perform exercises s uch as running, walking, and skipping for at least 30 minutes every day. Second visit on August 20, 2018: Height measured: $99.5 \mathrm{~cm}$, weight $14.5 \mathrm{~kg}$, and family members told the child that the amount of food consumed slightly increased, the amount of snacks decreased, the mood was more dry and irritable than before, and the night rest gradually became normal. Peel, red peony root, hemp kernel plus Ophiopogon japonicus, southern Adenophora, 28 doses. Children's massage and daily nursing program remain the same as before. Follow up after 3 months.

The third visit on December 8, 2019: Height: $101.5 \mathrm{~cm}$, weight 1 5kg, family members of the child complained: The diet has increased significantly, no partial eclipse, addiction to snacks, calm temperament, night rest, and normal bowel movements. The family members complained that the child had difficulty in feeding medications. Considering the safety of long-term medication, they suspended oral Chinese medicine and switched to the homemade spleen-tonifying ointment combined with pediatric massage in the Affiliated Hospital of Shaanxi University of Traditional Chinese Medicine, and insisted on exercising. Regular follow-up visits.

In the fourth consultation on April 8, 2020, the child is $111 \mathrm{~cm}$ tall and weighs $18.5 \mathrm{~kg}$. The child has no obvious symptoms. Regular outpatient follow-up visits.

Note: In this case, the child started to add complementary food in October, which was more than 4 months later than the normal supplementary food addition time of 4-6 months, which made the child insensitive to food and poor in chewing function during infancy. The diet mainly consists of beef and mutton. The child has been fond of eating meat since childhood. Due to long-term improper feeding, the syndrome of spleen deficiency and liver depression has formed. For a long time, the child has a lag in growth and development. The treatment focuses on strengthening the spleen and soothing the liver. Sijunzi Decoction is the core to invigorate the spleen and qi, add Jiao Sanxian to eliminate stagnation, and use Bupleurum, Cyperus rotundus, Danpi, and red peony root to clear heat and nourish blood and soothe the liver. In addition, children suffer from constipation and insomnia. Add hemp seed and jujube seed to treat the symptoms. At the second visit, the symptoms of the child were significantly reduced, but the course of the disease was longer to prevent the liver from becoming stagnant and change the fire and yin. After using Ophiopogon japonicus, Radix Adenophora and other products for clearing heat and nourishing yin, the child at the third visit had no obvious discomfort. During this period, the function of spleen transportation and transformation has tended to recover. The decoction is stopped and the spleen-tonifying ointment is used in our hospital. At the same time, it cooperates with the growth and development of children in this hospital and the diet and life regulation. The whole diagnosis and treatment process uses the spleen as the core of treatment. The spleen can nourish the whole body and eventually promote the growth and development of children. 


\section{Conclusion}

Although the cause of ISS is not yet clear, and there is no clear clinical guideline to include growth hormone in the treatment of ISS, ISS not only affects the height of children, but also causes psychological and social problems. With the progress of science and the development of the times, more and more parents pay attention to ISS. Through the clinical experience of Chinese medicine in the diagnosis and treatment of ISS in the past 10 years, Mr. Zhang has applied the theory of "spleen-liver and kidney" to the diagnosis and treatment of children with ISS. The treatment of spleen is to soothe the liver and nourish the kidney, that is, to invigorate the spleen to achieve the harmony and mutual use of the spleen, liver, and kidney, promote each other, and ultimately promote the growth and development of children. The medication is simple but not simple. The hope is the treatment of ISS provides new ideas.

\section{References}

[1] Endocrinology, Genetics and Metabolism Group of Pediatrics Branch of Chinese Medical Association. Guidelines for diagnosis and treatment of short stature children [J]. Chinese Journal of Pediatrics, 2008, 46(6): 428-430.

[2] Liu Fang, Shen Linghua, Wei Haiyan, Chen Yongxing. (2019). Research progress of idiopathic short stature [J]. Clinical Convergence, 2019, 34(10): 875-879.

[3] Xu Lu, Xiao Yanfeng, Wang Yaping, Huang Junpeng. (2019). Sleeping, diet and behavior problems in children with short stature A case-control study [J]. Chinese Journal of Children’s Health, 2019: 74-78.

[4] Zhou Shasha, Li Bian. (2020). Application range and side effects of growth hormone [J]. China Journal of Children's Health, 2020, 27: 24-27.

[5] Li Hui. (2019). Short stature and the standardized use of growth hormone [J]. Chinese Journal of Practical Pediatrics, 2019, 34(10): 815-818.

[6] Wu Junyan. (2016). Theory of Kidney Brain Xiangguan in Traditional Chinese Medicine Research [D]. 2016, 31-34.

[7] Lin Xiaoyuan, Cai Guangxian, Tan Yuansheng. (2015). Discussion on the theory of "spleen and kidney correlation" from the brain-gut axis [J]. Shizhen Traditional Chinese Medicine and Materia Medica, 2015, 26(9): 2210-2211.

[8] Xiao Shiju, Ji Yunrun, Xian Fuyang, Zhang Guangzhong. (2019). The relationship between the intestinal brain theory and the theory of "thinking about hurting the spleen" in traditional Chinese medicine [J]. Xinglin Traditional Chinese Medicine, 2019, 39(9): 1128-1131.

[9] Sun Shuangxi, Bai Xiaoxin. (2017). Discussing the understanding of the brain-gut axis from the perspective of TCM doctors' theory and pathology [J]. Shaanxi Journal of Traditional Chinese Medicine, 2017, 38(6): 787-788.

[10] Zhou Sufang, Li Yifeng, Wang Min. (2015). The theory of intestine-liver axis and the relationship between liver and spleen [J]. Journal of Traditional Chinese Medicine, 2015, 55(4): 352-357.

[11] Zhang Qi, Liu Changyun, Zhu Hailing, Li Xiuming, Zhai Yuping, Zheng Yang. (2018). Clinical causes of short growth in children Multivariate analysis [J]. Journal of Weifang Medical College, 2018, 40(1): 40-44.

[12] Zhang Chan, Li Qiang, Hu Mingyue, Wang Yongxin. (n.d.). The influence of parental height genetic factors on children’s height $[\mathrm{J}]$. 